Original Article

\title{
Factors inducing falling in schizophrenia patients
}

Yoko Tsuji, OTR, MS ${ }^{1 *}$, Yoshiteru Akezaki, RPT, $\mathrm{PhD}^{2)}$, Kohei Mori, $\mathrm{RPT}^{1)}$, Yoshimi Yuri, OTR, MS ${ }^{1}$, Hitomi Katsumura, OTR ${ }^{3)}$, Tomihiro Hara, OTR ${ }^{3)}$, Yuki Usui, OTR ${ }^{3)}$, Yoritaka Fujino, OTR ${ }^{3)}$, Takuo Nomura, RPT, PhD ${ }^{1)}$, Fumio Hirao, MD ${ }^{3)}$

1) Division of Occupational Therapy, Department of Rehabilitation Sciences, Faculty of Allied Health Sciences, Kansai University of Welfare Sciences: 3-11-1 Asahigaoka, Kashiwara-City, Osaka 582-0026, Japan

2) Department of Rehabilitation, National Hospital Organization Shikoku Cancer Center, Japan

3) Ueno Hospital, General Incorporated Foundation Shigisan Hospital, Japan

\begin{abstract}
Purpose] The purpose of this study is to investigate the factors causing falling among patients with schizophrenia hospitalized in psychiatric hospitals. [Subjects and Methods] The study subjects were divided into either those having experienced a fall within the past one year (Fall group, 12 patients) and those not having experienced a fall (Non-fall group, 7 patients), and we examined differences between the two groups. Assessment items measured included muscle strength, balance ability, flexibility, body composition assessment, Global Assessment of Functioning scale (GAF), the antipsychotic drug intake, and Drug Induced Extra-Pyramidal Symptoms Scale (DIEPSS). [Results] As a result, significant differences were observed in regard to One leg standing time with eyes open, Time Up and Go Test (TUGT), and DIEPSS Sialorrhea between the Fall group and the Non-fall group. [Conclusion] These results suggest that a decrease in balance ability was significantly correlated with falling in schizophrenia patients.

Key words: Schizophrenia, Balance, Falls
\end{abstract}

(This article was submitted Oct. 27, 2016, and was accepted Nov. 24, 2016)

\section{INTRODUCTION}

According to the Patient Survey of 2005 carried out by the Ministry of Health, Labour and Welfare, Japan ${ }^{1)}$ of 326,000 patients hospitalized due to "Mental and Behavioral Disorders," approximately 43.5\%, i.e., approximately 142,000 patients, were elderly persons aged 65 years or older. Moreover, according to the Patient Survey of 20112) of 282,000 hospitalized patients, approximately $48.2 \%$, i.e., approximately 136,000 patients, were 65 years of age or older. While the patients in psychiatric hospitals are aging, a tendency has been observed for the duration of hospitalization to be as long as 296 days ${ }^{2}$ limiting the opportunities for patients to act of their own accord.

Recently, the rate of falling is high for hospitalized patients in psychiatric hospitals with falling being the most frequently occurring issue when it comes to managing medical safety ${ }^{3)}$. Regarding the relationship between falling and drugs in nursing home residents, while it has been reported that the risk of falling increases as the psychotropic dosage increases ${ }^{4}$, or that the risk of falling for patients taking tricyclic antidepressant drugs is approximately three times higher than that for those not taking psychotropic drugs ${ }^{5)}$, some negative reports exists as well ${ }^{6}$, and the debate on this is still controversial. However, there are few reports that showed relationship between falling and physical function in patients with schizophrenia. Regarding the relationship between falling and physical function, physical factors in addition to environmental factors are noted in

*Corresponding author. Yoko Tsuji (E-mail: tsuji@tamateyama.ac.jp)

(C2017 The Society of Physical Therapy Science. Published by IPEC Inc.

This is an open-access article distributed under the terms of the Creative Commons Attribution Non-Commercial No Derivatives (by-nc-nd) License $<$ http://creativecommons.org/licenses/by-nc-nd/4.0/>. 
elderly persons without mental disorders; specifically, a decrease in muscle strength of the lower limbs, walking ability, and balance ability are seen as problematic ${ }^{7}$. After falling, it is noted that activities of daily living (ADL) deteriorate due to bone fractures, inducing limited activity caused by post-fall syndrome, losing confidence, developing symptoms of depression or anxiety, and decreasing the sphere of action due to the fear of falling ${ }^{8,9)}$. Since the daily life activities of patients are further limited after falling, it is important to prevent mental disorder patients from falling during hospitalization.

In the present study, we evaluated patients with schizophrenia hospitalized in psychiatric hospitals with the objective of analyzing the factors causing falling.

\section{SUBJECTS AND METHODS}

The study subjects included 19 patients with schizophrenia (male: 8 patients, female: 11 patients, mean \pm SE age: $63.3 \pm$ 6.1 years) hospitalized in a psychiatric hospital. All patients were admitted to the psychiatric rehabilitation ward. The exclusion criteria were patients using a wheelchair, those with mental disorders too severe to allow them to understand the study explanation, and those whose ADL was decreasing due to orthopedic disorders such as spinal cord injuries.

Consent to participate in the study was obtained from the subjects upon providing a verbal explanation about the study purport and study content. The study was carried out with the approval of the Research Ethics Committee of Kansai University of Welfare Sciences (Approval No. 14-15).

Assessment items measured included muscle strength (grip strength, and 30-second chair stand test ${ }^{10)}$ ), balance ability (One leg standing time with eyes open/closed, Functional Reach Test [FRT ${ }^{11)}$ ], and TUGT ${ }^{12}$ ), flexibility (long sitting position toe touching distance $\left.{ }^{13}\right)$, walking ability $\left(10 \mathrm{~m}_{\text {maximum }}\right.$ walking speed $\left.{ }^{14)}\right)$, sleeping condition (Athens insomnia scale ${ }^{15}$ ), Global Assessment of Functioning scale $(\mathrm{GAF})^{16)}$, the antipsychotic drug intake, Drug Induced Extra-Pyramidal Symptoms Scale (DIEPSS) ${ }^{17)}$, and number of falls.

Grip strength of the dominant hand was measured using GRIP-D (Takei Scientific Instruments Co., Ltd.).

The One leg standing time with eyes open/closed was measured for the pivotal foot, standing bare foot with both hands at the waist and elevating one foot, with 60 seconds set as the maximum.

FRT was measured with both hands in accordance with the method reported by Tsushima et al. ${ }^{18)}$, using a reach measuring instrument (OG Wellness Technologies Co., Ltd.).

Long sitting position toe touching distance was measured using EKJ09 (EVERNEW Inc.), in accordance with the measuring methods of the New Physical Fitness Tests by the Ministry of Education, Japan Culture, Sports, Science and Technology.

Grip strength, the 30-second chair stand test, One leg standing time with eyes open/closed, FRT, TUGT, long sitting position toe touching distance, and $10 \mathrm{~m}$ maximum walking speed were each measured twice, with the maximum and fastest values adopted as representative values.

Regarding the antipsychotic drug intake, the average dosage from one month before measurement day was extracted using the chlorpromazine-equivalent dosage.

A fall was defined as "when body parts other than the sole of the foot touched the floor/ground, against ones will" based on the definition by Gibson ${ }^{19)}$, with patients having experienced a fall at least once during the past 1 year assigned to the Fall group, while those not having experienced a fall were assigned to the Non-fall group. In addition, we investigated the fall situation using the "incident and accident reports".

Regarding the statistical analyses, a Mann-Whitney U-test and $\chi^{2}$ test were used to evaluate the differences in gender, age, BMI, complication, muscle strength, balance ability, flexibility, walking ability, sleeping condition, GAF, the antipsychotic drug intake, and DIEPSS between the Fall group and the Non-fall group. Statistical analysis was performed using IBM SPSS statistics 22.0. The statistical significance level was set at $<5 \%$.

\section{RESULTS}

A total of 12 patients (male: 4 patients, female: 8 patients, mean \pm SE age: $59.1 \pm 15.5$ years) were included in the Fall group while 7 patients (male: 4 patients, female: 3 patients, mean \pm SE age: $61.3 \pm 7.1$ years) were included in the Non-fall group.

The results of a univariate analysis are provided in Table 1. Significant differences were observed for One leg standing time with eyes opened, TUGT, and DIEPSS Sialorrhea between the Fall group and the Non-fall group $(\mathrm{p}<0.05)$.

\section{DISCUSSION}

In the present study, the presence or absence of having experienced a fall in patients with schizophrenia along with their muscle strength, balance ability, flexibility, walking ability, sleeping condition, GAF, and the antipsychotic drug intake were evaluated.

Regarding the physical function factors affecting falling, they reported that lower limbs muscle weakness ${ }^{20-22)}$ and decreased balance ability ${ }^{20,21)}$ are the factors involved in the elderly without mental disorders and stroke patients. In addition, 
Table 1. The comparison between factors in fall group and Non-fall group

\begin{tabular}{|c|c|c|}
\hline Item & Fall group $(\mathrm{n}=12)$ & Non-fall group $(n=7)$ \\
\hline Gender (male/female) & $5 / 7$ & $4 / 3$ \\
\hline Age (yrs) & $64.5 \pm 5.4(53-72)$ & $61.3 \pm 7.1(54-74)$ \\
\hline BMI $\left(\mathrm{kg} / \mathrm{m}^{2}\right)$ & $22.4 \pm 2.7$ & $23.2 \pm 3.7$ \\
\hline \multicolumn{3}{|l|}{ Complication } \\
\hline Diabetes mellitus (normal/ abnormal) (n) & $(10 / 2)$ & $(7 / 0)$ \\
\hline Hyperlipidemia (normal/ abnormal) (n) & $(9 / 3)$ & $(7 / 0)$ \\
\hline Hypertension (normal/ abnormal) (n) & $(9 / 3)$ & $(7 / 0)$ \\
\hline Grip strength (kg) & $18.0 \pm 6.6$ & $26.8 \pm 10.9$ \\
\hline 30-s chair stand test (times) & $12.2 \pm 2.7$ & $16.4 \pm 7.5$ \\
\hline One leg standing time with eyes open (seconds) & $4.7 \pm 6.2$ & $19.9 \pm 21.5^{*}$ \\
\hline One leg standing time with eyes close (seconds) & $1.8 \pm 2.8$ & $3.2 \pm 2.7$ \\
\hline Functional Reach Test (cm) & $20.2 \pm 10.6$ & $22.3 \pm 9.5$ \\
\hline Time Up and Go Test (seconds) & $8.7 \pm 1.6$ & $6.6 \pm 1.0^{*}$ \\
\hline Long sitting position toe touching distance $(\mathrm{cm})$ & $26.0 \pm 7.5$ & $26.1 \pm 16.9$ \\
\hline 10 m maximal walking speed (m/min) & $7.4 \pm 2.3$ & $5.9 \pm 1.4$ \\
\hline Athens insomnia scale (scores) & $4.6 \pm 5.8$ & $5.0 \pm 2.8$ \\
\hline GAF (scores) & $30.6 \pm 9.0$ & $37.0 \pm 10.5$ \\
\hline Antipsychotic drug doses (mg) & $545.4 \pm 491.3$ & $481.4 \pm 697.4$ \\
\hline DIEPSS: Gait & $2.1 \pm 0,8$ & $1.4 \pm 1.0$ \\
\hline Bradykinesia & $1.8 \pm 0.9$ & $1.4 \pm 0.8$ \\
\hline Sialorrhea & $1.1 \pm 0.9$ & $0.1 \pm 0.4^{*}$ \\
\hline Muscle rigidity & $1.0 \pm 1.0$ & $0.7 \pm 0.8$ \\
\hline Tremor & $1.0 \pm 1.0$ & $0.4 \pm 0.8$ \\
\hline Akathisia & $0.6 \pm 0.7$ & $0.4 \pm 0.8$ \\
\hline Dystonia & $0.5 \pm 1.0$ & $0 \pm 0$ \\
\hline Dyskinesia & $0.8 \pm 0.6$ & $0.1 \pm 0.4$ \\
\hline Overall severity & $1.8 \pm 0.9$ & $1.4 \pm 0.8$ \\
\hline
\end{tabular}

$* \mathrm{p}<0.05$ : significant difference between the groups; Athens insomnia scale: $0-24$ scores; GAF: $0-100$ scores; DIEPSS: 0-4 rates (0: Normal, 1: Minimal, 2: Mild, 3: Moderate, 4: Severe)

Ikai et al. ${ }^{22)}$ pointed out that there is a relationship between falling and dynamic balance ability, so it is useful to evaluate the dynamic balance ability in order to predict falling. Furthermore, it is reported that the balance exercise was useful in fall prevention ${ }^{23)}$. Based thereon, it is inferred that in subjects without mental disorders, falling is strongly associated with the physical functions of lower limb muscle strength and balance ability. As a result of the present study, since the static and dynamic balance ability in the Fall group was lower than the Non-fall group, it was suggested that a decrease in balance ability has a strong effect as a factor inducing falling in mental disorder patients as well.

Regarding the relationship between drugs and falling, drugs are involved in falling, and for psychotropic drugs in particular, which act on the central nerve system, it has been reported that psychotropic drugs increase the risk of falling/tumbling ${ }^{24)}$. Moreover, it has been indicated that the risk of falling/tumbling further increases in patients taking multiple psychotropic drugs $^{4}$. At the same time, Shigeyama et al. reported that while they evaluated the relationship between the antipsychotic drug dosage and falls/tumbles based on the results that the number of falls/tumbles was significantly higher when antipsychotic drugs were administered, there was no obvious relationship observed between them ${ }^{25}$. In the present study, the antipsychotic drug dosage did not demonstrate any significant differences between the Fall group and the Non-fall group. The reason for the low involvement of antipsychotic drug dosage was suspected to be due to the fact that antipsychotic drugs are prescribed by physicians only after assessing ADL and physical function/ability, in addition to mental function, assuming there is no negative impact on the assessed items.

Lastly, there were some limitations in the present study. This was a cross-sectional study and we believe that the effect of decreasing the number of falls by improving balance ability can be determined by follow-up or intervention studies, which will require further evaluation. 


\section{REFERENCES}

1) Japanese Ministry of Health, Labour and Welfare (2006): The 2005 Briefing Survey on Patient. http://www.mhlw.go.jp/ toukei/saikin/hw/kanja/05/toukei03. html (Accessed Sep. 22, 2015).

2) Japanese Ministry of Health, Labour and Welfare. (2012). The 2011 Briefing Survey on Patient. http://www.mhlw.go.jp/ toukei/saikin/ hw/kanja/11/d1/kanja. pdf\#search (Accessed Sep. 22, 2015)

3) Hosoi T, Makino E: A survey on falling accident cases of patients in psychiatric wards in Japan. Japanese Journal of Psychiatric of Psychiatric Rehabilitation, 2008, 12: 55-62 (in Japanese).

4) Cooper JW, Freeman MH, Cook CL, et al.: Assessment of psychotropic and psychoactive drug loads and falls in nursing facility residents. Consult Pharm, 2007, 22: 483-489. [Medline] [CrossRef]

5) Thapa PB, Gideon P, Fought RL, et al.: Psychotropic drugs and risk of recurrent falls in ambulatory nursing home residents. Am J Epidemiol, 1995, 142: 202-211. [Medline]

6) Landi F, Onder G, Cesari M, et al. Silver Network Home Care Study Group: Psychotropic medications and risk for falls among community-dwelling frail older people: an observational study. J Gerontol A Biol Sci Med Sci, 2005, 60: 622-626. [Medline] [CrossRef]

7) Yoshikawa Y, Kajita H, Matsuda K, et al.: Comparison of fall-related factors of the elderly with good or poor motor function: prospective study using logistic regression analysis. J Phys Edu Med, 2013, 14: 9-16.

8) Kannus P, Sievänen H, Palvanen M, et al.: Prevention of falls and consequent injuries in elderly people. Lancet, 2005, 366: 1885-1893. [Medline] [CrossRef]

9) Alarcón T, González-Montalvo JI, Otero Puime A: [Assessing patients with fear of falling. Does the method use change the results? A systematic review]. Aten Primaria, 2009, 41: 262-268. [Medline]

10) Jones CJ, Rikli RE, Beam WC: A 30-s chair-stand test as a measure of lower body strength in community-residing older adults. Res Q Exerc Sport, 1999, 70: 113-119. [Medline] [CrossRef]

11) Weiner DK, Duncan PW, Chandler J, et al.: Functional reach: a marker of physical frailty. J Am Geriatr Soc, 1992, 40: 203-207. [Medline] [CrossRef]

12) Podsiadlo D, Richardson S: The timed "Up \& Go": a test of basic functional mobility for frail elderly persons. J Am Geriatr Soc, 1991, 39: 142-148. [Medline] [CrossRef]

13) Japanese Ministry of Education: Culture, Sports, Science and Technology (1999): The New Physical Fitness Tests Protocol. http://www.mext.go.jp/a_menu/ sports/stamina/05030101/003.pdf (Accessed Aug. 15, 2014)

14) Suzuki K, Imada G, Iwaya T, et al.: Determinants and predictors of the maximum walking speed during computer-assisted gait training in hemiparetic stroke patients. Arch Phys Med Rehabil, 1999, 80: 179-182. [Medline] [CrossRef]

15) Okajima I, Nakajima S, Kobayashi M, et al.: Development and validation of the Japanese version of the Athens Insomnia Scale. Psychiatry Clin Neurosci, 2013, 67: 420-425. [Medline] [CrossRef]

16) American Psychiatric Association: Diagnostic and Statistic Manual of Mental Disorders (DSM-IV). Washington DC: APA, 1994.

17) Kim JH, Jung HY, Kang UG, et al.: Metric characteristics of the drug-induced extrapyramidal symptoms scale (DIEPSS): a practical combined rating scale for drug-induced movement disorders. Mov Disord, 2002, 17: 1354-1359. [Medline] [CrossRef]

18) Tsushima H, Tsushima E, Tsushima K, et al.: How does the functional reach change with age in healthy people? Bull Health Sci Hirosaki, 2006 , 5: 166-172.

19) Gibson MJ: Improving the health of older people. Kane RL, Evans JG, Macfadyen D (eds.), In: a World View. New York: Oxford Univ Press, 1990, pp 296-315.

20) Muir SW, Berg K, Chesworth B, et al.: Use of the Berg Balance Scale for predicting multiple falls in community-dwelling elderly people: a prospective study Phys Ther, 2008, 88: 449-459. [Medline] [CrossRef]

21) Gunter KB, White KN, Hayes WC, et al.: Functional mobility discriminates nonfallers from one-time and frequent fallers. J Gerontol A Biol Sci Med Sci, 2000, 55: M672-M676. [Medline] [CrossRef]

22) Ikai T, Kamikubo T, Takehara I, et al.: Dynamic postural control in middle-aged and elderly people. Jpn J Rehabil Med. 2002, 39: 311-316. [CrossRef]

23) Province MA, Hadley EC, Hornbrook MC, et al.: The effects of exercise on falls in elderly patients. A preplanned meta-analysis of the FICSIT Trials. Frailty and injuries: cooperative studies of intervention techniques. JAMA, 1995, 273: 1341-1347. [Medline] [CrossRef]

24) Souchet E, Lapeyre-Mestre M, Montastruc JL: Drug related falls: a study in the French Pharmacovigilance database. Pharmacoepidemiol Drug Saf, 2005, 14: 11-16. [Medline] [CrossRef]

25) Shigeyama M, Taguchi M, Maeyama N, et al.: Study on relationship between psychotropic medications and risk of falls. JSPHCS, 2011, 37 : 49-55. 